\section{Autobranche im Nachhaltigkeitsstau?}

\section{Nach der ersten Umweltbewertung der Automobilindustrie vom Januar 1999 hat die oekom re- search AG nun ein Corporate Responsibility Rating der Branche vorgelegt. Dabei wurde die Un- tersuchung um eine Bewertung der Sozial- und Kulturverträglichkeit erweitert. Die Ergebnisse sind kein Ruhmesblatt.}

I

Von Dirk Reinhard $m$ bisherigen Öko-Rating wurden die Bereiche Umweltmanagement, Umweltverträglichkeit der Fahrzeuge sowie Ressourcenverbräuche und Emissionen im Produktionsprozess untersucht. Neu hinzugekommen sind nun das Sozialmanagement, das Verhältnis zu den Mitarbeitern, sowie das Verhältnis zu externen Anspruchsgruppen. Abbildung 1 gibt Beispiele für die einzelnen Untersuchungskriterien.

Bei allen Kriterien liegt ein Schwerpunkt der Untersuchung darin, herauszufinden ob das Unternehmen möglichst gleichwertige Standards weltweit anwendet. Die Untersuchung stïtzt sich neben der

\begin{tabular}{|l|l|}
\hline \multicolumn{3}{|c|}{ Abb. l: Ausgewählte neue Einz } \\
\hline Sozialmanagement: & \multicolumn{1}{|c|}{ Mitarbeiter: } \\
\hline - Unternehmensleitbild und -ziele & Mitbestimmung \\
\hline - Beauftragte für Personal, & - Entlohnung \\
\hline soziale Fragen, Verhaltens- & - Arbeitszeiten \\
\hline richtlinien, Unternehmensleit- & - Sicherheit \\
\hline bilder etc. & Gesundheit \\
\hline - Sozialaudits (z.B. zur & - Schulung \\
\hline Einhaltung von Standards, & - Humanisierung \\
\hline Mitarbeiterzufriedenheit etc.) & des Arbeitsplatzes \\
\hline - Programm & - Förderung von \\
\hline - Sozialberichterstattung & benachteiligten \\
\hline & Gruppen \\
\hline & \\
\hline
\end{tabular}

Auswertung eines umfangreichen Fragebogens auch auf zahlreiche externe Quellen wie z.B. Corporatewatch, Labournet oder Transparency International (1).

Insgesamt war die Untersuchung von einer sehr zurückhaltenden Teilnahmebereitschaft im Bereich der Bewertung der Sozial- und Kulturverträglichkeit gekennzeichnet. So waren von den 19 angesprochenen Unternehmen allein BMW, DaimlerChrysler und General Motors bereit, die ge- stellten Fragen zu beantworten. Wenn auch einige Unternehmen großes Verständnis für die Untersuchung hatten, so wurde die Ablehnung in der Regel damit begründet, dass ein zentralisiertes Berichtswesen über die relevanten Informationen fehle.

Dieses Verhalten ist in Anbetracht der Vorkommnisse bei Unternehmen wie Mitsubishi und Ford, die lange Zeit Sicherheitsbedenken zurückgehalten haben bzw. gesetzlichen Berichtspflichten nicht nachgekommen sind, sehr bedenklich. In Europa sind beinahe alle deutschen Hersteller ins Visier der Kartellbehörden geraten. Einzig BMW hat damit bislang keine Probleme.
Mit steigenden Ölpreisen ist auch der Anreiz, sparsame Autos zu bauen, gestiegen. Dies wurde daran deutlich, dass zunehmend Modelle auf den Markt kommen, die vier oder gar nur drei Liter pro 100 Kilometer verbrauchen. So wird der Drei-Liter-Lupo von VW bald Gesellschaft von Audi bekommen. Opel hat einen Vier-Liter-Astra auf dem Markt.

Dennoch: Die größten Verbesserungspotenziale sind immer noch im Bereich der Fahrzeuge selbst vorhanden. Neue Richtlinien zur Vermeidung von Schadstoffen wie Schwermetallen im Fahrzeug stellen nicht nur die Hersteller, sondern auch Zulieferer vor neue Herausforderungen.

Dringender Handlungsbedarf besteht immer noch beim Spritverbrauch. Gerade die amerikanischen Hersteller produzieren jährlich noch Millionen von überdimensionierten und spritfressenden Geländefahrzeugen und Pickups, die zudem noch aufgrund von Sicherheitsmängeln ins Gerede gekommen sind. Bedenklich ist zudem, dass diese Fahrzeuge häufig unnötig schwer sind, damit sie steuerlich als Lastwagen anerkannt werden und somit beispielsweise nur noch die niedrigeren Grenzwerte für LKW-Emissionen einhalten müssen. Doch auch in Deutschland steht, wie etwa die Werbung zeigt, Freude am Fahren vielfach noch vor Umweltschutz.

\section{Die Gesamtergebnisse}

Insgesamt betrachtet haben im Umweltbereich Audi und VW ihre guten Positionen behauptet und führen das Ranking mit Platz eins und zwei an, gefolgt von Renault. Alle drei Unternehmen erzielten ein B-. Am Ende der Skala liegen die japanischen Unternehmen Mitsubishi (C-) und Mazda (D+). Im Social/ Cultural Rating sowie in der Gesamtbewertung des Corporate Responsibility-Ratings erzielte BMW mit einem B- den ersten Platz.

Insgesamt geht es im Umweltschutz der Automobilbranche langsam aufwärts. Bezüglich der Teilnahmebereitschaft zur Veröffentlichung von Sozialdaten ist allerdings noch keine Trendwende abzusehen.

\section{Anmerkung}

(1) Vgl. zum Konzept des Ratings auch die Artikel „Neues Nachhaltigkeitsrating" in Ökologisches Wirtschaften 2/2000, S. 28, und "Ethik mischt mit" in punkt.um 9/00, S. 8-9. bereich. Hier ließen sich immerhin 14 Unternehmen in die Karten schauen. Die Untersuchung der Umweltschutzaktivitäten hat gezeigt, dass die meisten Verbesserungen im Umweltschutz in den vergangenen eineinhalb Jahren im Bereich Umweltmanagement und Umweltdaten, etwa hinsichtlich des Energie- und Wasserverbrauchs in der Produktion, erzielt wurden. So haben gerade die französischen Hersteller hier stark aufgeholt. 
(c) 20I0 Authors; licensee IÖW and oekom verlag. This is an article distributed under the terms of the Creative Commons Attribution Non-Commercial No Derivates License (http://creativecommons.org/licenses/by-nc-nd/3.o/), which permits unrestricted use, distribution, and reproduction in any medium, provided the original work is properly cited. 